
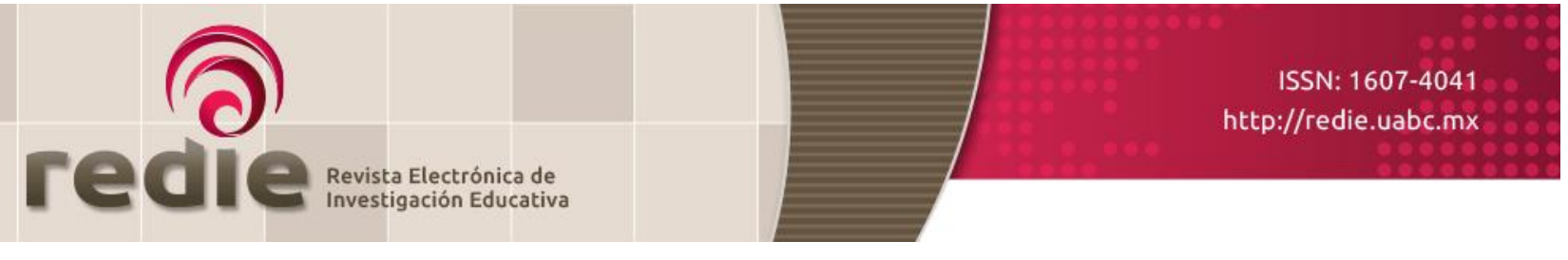

Vol. 19, Núm. 2, 2017

\title{
Autoconcepto físico y académico en niños de contextos marginados en México
}

\section{Physical and Academic Self-Perspective in Children from Marginalized Contexts in México}

\author{
Martha Leticia Gaeta González (*) marthaleticia.gaeta@upaep.mx \\ Judith Cavazos Arroyo (*) judith.cavazos@upaep.mx \\ (*) Universidad Popular Autónoma del Estado de Puebla \\ (Recibido: 21 de enero de 2014; Aceptado para su publicación: 4 de noviembre de 2015) \\ Cómo citar: Gaeta, M. L. y Cavazos, J. (2017). Autoconcepto físico y académico en niños de contextos marginados en México. Revista \\ Electrónica de Investigación Educativa, 19(2), 114-124. https://doi.org/10.24320/redie.2017.19.2.604
}

\section{Resumen}

Esta investigación examinó el autoconcepto físico y académico y su relación con algunas variables socioculturales, como son: la alimentación, la actividad física, así como la preocupación por la obesidad y la apariencia física en 500 niños de 5o. y 60. grado de educación primaria. Se desarrolló una investigación no experimental, descriptiva y transversal en dos contextos marginados en México. Se realizaron análisis de conglomerados a fin de identificar perfiles de estudiantes, de acuerdo con las variables socioculturales de estudio. Los resultados evidenciaron, en general, autoconceptos físicos y académicos bajos, aunque algunas diferencias significativas entre géneros. Asimismo, el análisis de conglomerados arrojó dos grupos: uno más consciente y otro menos consciente del valor y nivel nutritivo de los alimentos que consumen en la tienda escolar.

Palabras clave: Identidad, alimentación escolar, pobreza.

\section{Abstract}

This study examined physical and academic self-perspective and how it relates to certain sociocultural variables, namely diet, physical exercise, and concern about obesity and physical appearance in $5005^{\text {th }}$ and $6^{\text {th }}$-grade elementary school students. A non-experimental, descriptive and cross-sectional study was carried out in two marginalized contexts in Mexico. Cluster analyses were performed to identify student profiles in accordance with the sociocultural variables of the study. The results show generally low levels of physical and academic self-perspective, despite some significant differences between the sexes. The cluster analysis also produced two groups: one with greater awareness and one with less awareness of the nutritional value of the food they eat from the school snack shop. 


\section{Introducción}

De manera reciente, en México se tomaron medidas alineadas con la Organización Mundial de la Salud (OMS) respecto a la promoción de una alimentación saludable, la realización de actividad física y otras medidas preventivas de salud entre distintos grupos poblacionales. Para el año 2010 se hicieron oficiales los lineamientos generales para el expendio o distribución de alimentos y bebidas en los establecimientos de consumo escolar de los planteles de educación básica, mismos que entraron en vigor el 1 de enero de 2011 para promover una alimentación y un entorno saludable, así como hábitos alimentarios para la prevención del sobrepeso y la obesidad infantil.

Aunado a la intervención del Estado, el propio contexto sociocultural, el acceso a la información, las nuevas formas de entretenimiento y la publicidad inciden en las percepciones, así como en el autoconcepto de los niños y adultos, bajo ciertos moldes y valores que se ejercitan en la cotidianidad (Costa, 2009). Con esta base se construyen formas particulares de subjetividades y ciudadanos, que a través de discursos y representaciones impactan el imaginario social, cuyo poder de influencia compite con la educación formal crítica (Bailón, 2013).

Dentro de los campos de la Educación y la Psicología se ha atendido el estudio del autoconcepto como un constructo psicológico útil en la comprensión de una amplia variedad de comportamientos (Mboya, 1999), incluyendo los hábitos saludables (Gosling, Stanistreet y Swami, 2008; Simms, Bock y Hackett, 2013). Sin embargo, aún es necesario seguir investigando las implicaciones del autoconcepto en el ámbito educativo en diferentes contextos (Yeung, Craven y Ali, 2013). Así, se plantearon dos objetivos para esta investigación: 1) examinar el autoconcepto físico y académico y su relación con algunas variables socioculturales, como son: la alimentación, la actividad física, así como la preocupación por la obesidad y la apariencia física en niños de 50. y 60. grado de educación primaria en dos contextos de alta marginación en México y, 2) identificar el número y las características distintivas de los conglomerados de estudiantes en relación a su alimentación, actividad física y preocupación por la obesidad y apariencia física.

El autoconcepto se refiere al conocimiento que poseen los individuos de las características o atributos que utilizan para definirse a sí mismos y al medio en el que se desenvuelven, diferenciándose de los demás (Franco, 2006). Engloba un amplio conjunto de imágenes, juicios y conceptos que el individuo tiene sobre sus distintos aspectos corporales, psicológicos, sociales y morales (Guillén, 2003). Algunos investigadores consideran que es más apropiado estudiar el autoconcepto desde sus dimensiones que hacerlo de forma genérica (Goñi, Ruíz de Azúa y Rodríguez, 2011); en este trabajo se han considerado dos de sus dominios: el físico y el académico.

El autoconcepto físico permite conocer las autodescripciones o autorrepresentaciones que una persona tiene de sí misma, reflejadas en la condición física, la competencia deportiva, el atractivo físico y la fuerza (Fox y Corbin, 1989; Tatlow-Golden y Guerin, 2010). El desarrollo del yo corporal en los infantes inicia con la autoconciencia en el segundo año de vida y continúa con el desarrollo del autoconcepto durante la etapa preescolar y más allá (Brownell, Zewars y Ramani, 2007). Un bajo autoconcepto puede minar la realización del pleno potencial humano de un niño haciéndolo susceptible a la estigmatización y poniéndose en riesgo su bienestar debido a la ansiedad y la depresión (Willows, Ridley, Eaine y Maximova, 2013).

Por otra parte, el autoconcepto académico se refiere a las autopercepciones académicas que tienen los estudiantes de sí mismos (Yeung et al., 2013), incidiendo en el bienestar psicológico, el comportamiento y los resultados educativos, como el rendimiento escolar y los logros (Chong, 2007; González, Leal, Segovia y Aracibia, 2012; Marsh y Craven, 2006). En relación con algunas variables demográficas se ha encontrado que el autoconcepto académico aumenta con la edad (Bolognini, Plancherel, Bettschart y Halfon, 1996) teniendo el nivel más bajo en la transición entre la escuela primaria y la secundaria, pero el autoconcepto físico disminuye con la edad (Lau, 1990). Las diferencias de género en el autoconcepto están sustentadas por trabajos empíricos en diferentes contextos. Los varones suelen presentar un mayor autoconcepto físico (Lau, 1990; Hay y Ashman, 2003) pero se ha evidenciado en las mujeres un mayor autoconcepto 
académico (Cairns, McWhirter, Duffy y Barry, 1990).

En México destacan más los estudios sobre autoconcepto físico y académico enfocados a muestras de adolescentes que de niños. El trabajo de Salum-Fares, Marín y Reyes (2011) encontró que tanto la dimensión física como académica explican menos del 20\% de la varianza del autoconcepto general en estudiantes de secundaria, pero Padilla, García y Suárez (2010) hallaron que la dimensión física es la que mayor relación muestra con las demás dimensiones del autoconcepto de los adolescentes. Otro estudio (Acosta y Gómez, 2003) evidenció que los estudiantes adolescentes mexicanos presentan altas tasas de insatisfacción con la imagen corporal, acentuándose más en las mujeres que en los hombres. En relación con el género, el estudio de Guido, Mújica y Gutiérrez (2011) identificó una sola diferencia significativa entre adolescentes masculinos y femeninos, encontrando que los hombres tienden a percibirse mejor que las mujeres sólo en el autoconcepto deportivo, es decir, se describen como más fuertes, atléticos y capaces de realizar ejercicios y actividades deportivas en comparación con el autoconcepto femenino.

La pobreza como condición social en México suele incluir rasgos como problemas familiares, hacinamiento habitacional, migraciones, separación de la familia, bajo acceso a servicios básicos como electricidad, agua potable y vías de comunicación, equipamiento mínimo en el hogar y limitaciones para cubrir las necesidades básicas de la familia (Ramírez, Devia y León, 2011). Estos aspectos involucran diversos factores de riesgo que llevan a resultados psicosociales negativos en la familia (Ramírez et al., 2011) y en los niños (Amar, 2000). Un estudio que comparó el autoconcepto de niños viviendo en situación de pobreza y aquellos que viven en un ambiente socioeconómico más favorecido encontró que es más bajo en los primeros, aunque el autoconcepto académico resultó bajo en ambos grupos de estudio (Alexis, 2014). Por su parte, otro estudio con niños provenientes de familias con riesgo psicosocial medio, caracterizado por situaciones de desventaja socioeconómica (Torres y Rodrigo, 2014), indicó que éstos presentan un autoconcepto más bajo y son más vulnerables a comportamientos disruptivos que pueden afectar su desempeño escolar y desarrollo personal.

\section{Método}

Se desarrolló una investigación no experimental, descriptiva y transversal (Kerlinger y Lee, 2002). El estudio se aplicó en dos juntas auxiliares del municipio de Puebla (México); la Resurrección y Santa María Xonacatepec. Ambas catalogadas como zonas de marginación y rezago entre las juntas a cargo del municipio de Puebla. Estas zonas presentan escasez de servicios públicos básicos, como calles pavimentadas, agua potable y seguridad (Páez, 2012). Como técnica para recolectar la información se utilizó la encuesta personal aplicada en los salones de clase, en el horario académico habitual, a través de un cuestionario autoadministrado dividido en secciones: alimentación, actividad física, preocupación por la obesidad y la apariencia física, y las escalas de Andrade y Pick (1986) de autoconcepto físico y autoconcepto como estudiante.

Los sujetos de estudio fueron estudiantes de 50. y 60. grado de cinco escuelas públicas de las dos juntas auxiliares, ubicados en tres turnos matutinos y dos vespertinos. Se aplicaron 500 encuestas durante el período del 7 al 14 de octubre de 2013, 490 cuestionarios fueron utilizables, ya que 10 de ellos fueron devueltos incompletos y se eliminaron a fin de obtener un análisis más preciso; los cuestionarios utilizables representan el $98 \%$ de los recibidos.

\section{Resultados}

El $51.4 \%$ de los entrevistados fueron niñas y $48.6 \%$ niños. $53.3 \%$ correspondieron al 50 . grado y $46.7 \%$ al 6o. grado de educación primaria. El rango de edad osciló entre los 9 y los 13 años, con un promedio de 10.6 años y una desviación estándar de 0.821 años (ver tabla I). 
Tabla I. Características de la muestra estudiada

\begin{tabular}{lrlll}
\hline Escuela $(\mathrm{n}=490)$ & $\mathrm{N}$ & Porcentaje & Sexo $(\mathrm{n}=490)$ & Porcentaje \\
\hline Escuela matutina B.J. & 92 & 18.8 & Niñas & 51.4 \\
Escuela vespertina B.J. & 120 & 24.5 & Niños & 48.6 \\
\cline { 5 - 6 } Escuela matutina J.L.P. & 72 & 14.7 & Grado & Porcentaje \\
\cline { 5 - 6 } Escuela matutina J.V. & 68 & 13.9 & 50. & 53.3 \\
Escuela vespertina M.H.C. & 138 & 28.2 & 6o. & 46.7 \\
\hline Edad (n=490) & $\mathrm{N}$ & Porcentaje & Encuestados por escuela/sexo (n=490) \\
\hline 9 & 31 & 6.3 & Escuela matutina B.J. & Niñas 21.4\% Niños 16\% \\
10 & 205 & 41.8 & Escuela vespertina B.J. & Niñas 24.2\% Niños 24.8\% \\
11 & 191 & 39.0 & Escuela J.L.P. & Niñas 13.5\% Niños 16\% \\
12 & 57 & 11.6 & Escuela matutina J.V. & Niñas 11.9\% Niños 16\% \\
13 & 6 & 1.2 & Escuela M.H.C & Niñas 29.0\% Niños 27.3\% \\
\hline
\end{tabular}

\subsection{Alimentación}

Con relación a la alimentación en la escuela, se encontró: la mayor parte (77.5\%) de las escuelas no cuenta con bebederos o garrafones de agua; la mayoría de los niños desayunan en casa (88.2\%); llevan a la escuela alimentos preparados desde el hogar (71.9\%), pero también la mayor parte de ellos (62.8\%) compra en la tienda escolar más de una vez a la semana. Los alimentos que más suelen traer de casa son tortas preparadas, agua, fruta y yogur, y los que suelen adquirir en la escuela son dulces, agua, tortas preparadas, agua de sabor, bolsas de frituras, frutas y chocolates (ver tabla II). El 55.8\% manifestó comprar en la tienda escolar una vez al día, $26.70 \%$ dos veces al día y $17.60 \%$ tres o más veces al día, pero $44.6 \%$ dijo comprar sólo algunos días de la semana, $23.1 \%$ sólo un día de la semana, $16.7 \%$ todos los días y $15.6 \%$ casi todos los días.

Tabla II. Alimentos que suelen llevarse de casa y los adquiridos en la escuela

\begin{tabular}{|c|c|c|c|c|c|}
\hline \multicolumn{3}{|l|}{ Desde casa } & \multicolumn{3}{|l|}{ Adquiridos en la escuela } \\
\hline Alimento & Frecuencia & Porcentaje & Alimento & Frecuencia & Porcentaje \\
\hline Torta & 293 & 31.74 & Dulces & 238 & 16.37 \\
\hline Agua simple o natural & 240 & 26.00 & Agua simple o natural & 160 & 11.00 \\
\hline Fruta & 119 & 12.89 & Tortas & 142 & 9.77 \\
\hline Yogurt & 103 & 11.16 & Agua de sabor & 117 & 8.05 \\
\hline Sándwich & 50 & 5.42 & Bolsa de Frituras (Frito Lay, Totis, etc.) & 111 & 7.63 \\
\hline Galletas & 39 & 4.23 & Frutas & 108 & 7.43 \\
\hline Golosinas & 30 & 3.25 & Chocolates & 103 & 7.08 \\
\hline Refresco & 28 & 3.03 & Chicharrines de harina & 88 & 6.05 \\
\hline Ensalada & 14 & 1.52 & Jugo & 84 & 5.78 \\
\hline Pizza & 3 & 0.33 & Galletas & 80 & 5.50 \\
\hline Frituras embolsadas & 1 & 0.11 & Chile en polvo & 59 & 4.06 \\
\hline Antojitos & 1 & 0.11 & Refresco & 42 & 2.89 \\
\hline Chicharrines & 1 & 0.11 & Leche de sabor & 29 & 1.99 \\
\hline Chocolates & 1 & 0.11 & Sincronizadas & 28 & 1.93 \\
\hline \multirow[t]{4}{*}{ Total } & 923 & 100 & Antojitos mexicanos & 24 & 1.65 \\
\hline & & & Panecillos o pasteles & 21 & 1.44 \\
\hline & & & Pizzas & 20 & 1.38 \\
\hline & & & Total & 1454 & 100 \\
\hline
\end{tabular}


En general, no existe una percepción positiva de los productos que se venden en la escuela; la mayor parte de los niños percibe los alimentos que se venden con bajo valor nutricional y el $45.7 \%$ los considera malos para la salud, aunque un $43.7 \%$ manifestó que no sabe si son buenos o malos para la salud (ver tabla III).

Tabla III. Percepción de los atributos nutricionales de los alimentos que venden en la escuela

\begin{tabular}{llcc}
\hline Nutrición & \multicolumn{1}{c}{ Percepción } & Frecuencia & Porcentaje \\
\hline Percepción del valor & Malo & 111 & 22.7 \\
nutritivo de los productos & No tan malo & 124 & 25.3 \\
que venden en la tienda & No tan bueno & 199 & 40.6 \\
escolar & Bueno & 56 & 11.4 \\
Importancia de los & Para nada importante & 105 & 21.4 \\
productos que vende la & No muy importante & 157 & 32.0 \\
tienda en una dieta sana & Algo importante & 114 & 23.3 \\
& Muy importante & 114 & 23.3 \\
Percepción de los & Buenos para la salud & 52 & 10.6 \\
productos que se venden & Malos para la salud & 224 & 45.7 \\
en la tienda escolar & No sabe & 214 & 43.7 \\
Calificación del nivel & Para nada nutritivo & 100 & 20.4 \\
nutricional de los productos & Casi no es nutritivo & 166 & 33.9 \\
que venden en la tienda & Un poco nutritivo & 190 & 38.8 \\
escolar & Muy nutritivo & 34 & 6.9 \\
\hline
\end{tabular}

\subsection{Actividad física y preocupación por la obesidad y la apariencia}

El 100\% respondió tener clase de Educación Física. El 90.6\% manifiesta practicar ejercicio adicional al que se realiza en la clase de Educación Física. De éstos, el 45.7\% practica más de dos veces a la semana, 28.3\% dos veces a la semana y el $26 \%$ una vez a la semana. $46.3 \%$ afirma practicar menos de una hora, $33.1 \%$ una hora y $20.6 \%$ más de una hora (ver tabla IV). En conversaciones informales, dos de las maestras comentaron que muchos de los niños hacen el recorrido casa-escuela-casa caminando, realizando recorridos de hasta 30 minutos, por lo que no necesariamente el ejercicio adicional consiste en la práctica de un deporte.

De acuerdo con la talla y peso proporcionados por los maestros de Educación Física, se encontró que respecto a los alumnos de 5o. año, los niños pesan en promedio 31.52 kilogramos (DT=6.82) y miden en promedio $133 \mathrm{~cm}$ (DT=7.11); mientras que las niñas pesan en promedio 31.63 kilogramos (DT=5.91) y miden en promedio $135 \mathrm{~cm}$ (DT=7.47). De acuerdo con la Academia Mexicana, un niño de 10 años debe pesar en promedio 30 kilos, con una talla en cm de132 (+- 6\%), y una niña debe pesar 30.4 kilos, con una talla en cm de 133.5 (+-6 \%). Respecto a los alumnos de 6o. año, los niños pesan en promedio 33.29 (DT $=7.11$ ) y miden en promedio $135.68 \mathrm{~cm}$ (DT=7.23), mientras que las niñas pesan en promedio 34.46 kilogramos (DT=7.49) y miden en promedio $137.60 \mathrm{~cm}$ (DT=7.48). De acuerdo con la Academia Mexicana, un niño de 11 años debe pesar en promedio 33.1 kilos, con una talla en cm de 139 (+- 6\%) y una niña debe pesar 34.7 kilos, con una talla en cm de 141 (+-6 \%). En general, puede decirse que ambos grupos se encuentran en un rango aceptable de peso y talla.

Al cuestionar sobre la apariencia física, el $76.5 \%$ considera que sí importa, aunque no se evidenció asociación a la variable por género $\left(\mathrm{Xi}^{2}=1.305, p=0.241\right)$. En relación a la obesidad, se encontró interés por saber más sobre ésta y preocupación por caer en la condición. Los resultados de la prueba t que comparó niñas y niños, resultó ser significativa ( $p=0.001$, medido en escala del 1 al 7) para las dos poblaciones en relación a ambas variables (interés y preocupación), encontrándose que las niñas presentan mayor preocupación por ambas condiciones (Preocupación por la obesidad M niñas=5.74 DT=1.85, M niños=5.03, DT=2.10; interés en saber más $M$ niñas=5.84, D.T.=1.68, $\mathrm{M}$ niños=5.14, DT=1.97). 
Tabla IV. Frecuencia de clase de educación física y práctica de ejercicio adicional

\begin{tabular}{llrr}
\hline & & Frecuencia & Porcentaje \\
\hline Frecuencia con la que se tiene & Una vez a la semana & 195 & 39.8 \\
clase de educación física & Dos veces a la semana & 283 & 57.8 \\
& Más de dos veces a la semana & 8 & 1.6 \\
& Una vez al mes & 4 & 0.8 \\
\hline Practica ejercicio adicional & Sí & 444 & 90.6 \\
a la clase de educación física & No & 46 & 9.4 \\
\hline
\end{tabular}

\subsection{Autoconcepto físico y académico}

Autoconceptos físico y académico. Los resultados muestran una tendencia hacia un autoconcepto físico débil, gordo, bajo, feo, chico, inactivo y enfermo. Mientras que el autoconcepto académico tiende a representarse como flojo, lento, tonto, malo, burro, incumplido, desorganizado y atrasado (ver tablas $\vee \mathrm{y}$ $\mathrm{VI})$.

Tabla V. Autoconcepto físico y académico por género

\begin{tabular}{|c|c|c|c|c|c|c|c|c|c|}
\hline \multirow{2}{*}{$\begin{array}{l}\text { Autoconcepto físico } \\
\text { Adjetivo bipolar } \\
\text { medido de } 1 \text { a } 5\end{array}$} & \multicolumn{2}{|c|}{ Niñas } & \multicolumn{2}{|c|}{ Niños } & \multirow{2}{*}{$\begin{array}{l}\text { Autoconcepto académico } \\
\text { Adjetivo bipolar } \\
\text { medido de } 1 \text { a } 5\end{array}$} & \multicolumn{2}{|c|}{ Niñas } & \multicolumn{2}{|c|}{ Niños } \\
\hline & M & DT & M & DT & & M & DT & M & DT \\
\hline Fuerte(1)/Débil(5) & 3.29 & 1.18 & 3.78 & 1.17 & Estudioso(1)/flojo(5) & 3.87 & 1.03 & 3.53 & 1.25 \\
\hline & & 1.1 & 3.7 & & & & & & 1.41 \\
\hline Bajc & 2.79 & 1.49 & 3.05 & 1.6 & & & 9 & 14 & 2.28 \\
\hline Gua & 3.69 & 1.04 & 3.79 & 1.04 & (5) & 4.11 & 1. & 3.98 & 1.15 \\
\hline & 2.7 & 1.4 & 3.13 & & lo(5) & & & & 1.07 \\
\hline nactivo(5) & 3.88 & 1.18 & 4.00 & 1.21 & ncumplido(5) & 3.87 & 1.21 & 3.81 & 1.22 \\
\hline \multirow[t]{3}{*}{ Enfermo(1)/Sano(5) } & 1.93 & 1.24 & 1.59 & 1.01 & jador(5) & 1.90 & 0.928 & 2.23 & 1.22 \\
\hline & & & & & & 3.98 & 1.167 & 3.76 & 1.33 \\
\hline & & & & & Atrasado(1)/Adelantado(5) & 2.48 & 1.228 & 2.51 & 1.39 \\
\hline
\end{tabular}

Tabla VI. Representación visual de las tendencias de los autoconceptos físico y académico por género

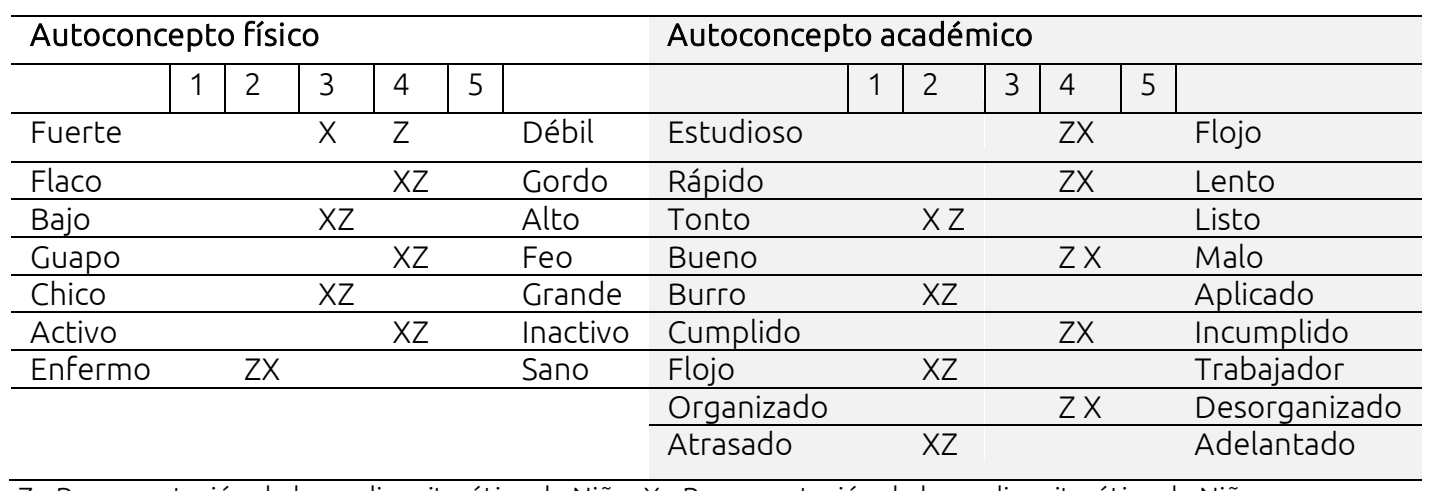

Z= Representación de la media aritmética de Niño, X= Representación de la media aritmética de Niña.

Nota: La cercanía de la respuesta a cada adjetivo (media aritmética) indica un mayor acuerdo con ese adjetivo.

Se realizó una prueba $t$ para dos muestras independientes para identificar si existían diferencias de autoconcepto físico y académico entre niños y niñas (ver tablas VII y VIII). Los resultados en cuanto al autoconcepto físico evidenciaron diferencias significativas respecto a fuerte(1)/débil(5) ( $a=.01>p=0.001)$, chico(1)/grande(5) ( $a=.01>p=0.004)$ y enfermo(1)/sano(5) $(a=.01>p=0.001)$, donde las niñas se perciben como más fuertes, más chicas y más sanas con respecto a los niños. En relación al autoconcepto académico, se encontraron diferencias significativas en estudioso(1)/flojo(5) $(a=.01>p=0.001)$, flojo(1)/trabajador(5) ( $a=.01>p=0.001)$ y marginalmente en organizado(1)/desorganizado(5) ( $p=0.054)$. 
Los niños se perciben más estudiosos, un poco más trabajadores y más organizados que las niñas.

Tabla VII. Resultados de prueba t para autoconcepto físico entre niños y niñas

\begin{tabular}{lccc}
\hline $\begin{array}{l}\text { Autoconcepto } \\
\text { Físico }\end{array}$ & $t$ & gl & $\begin{array}{c}\text { Sig. } \\
\text { (bilateral) }\end{array}$ \\
\hline Fuerte/Débil & -4.692 & 486.778 & $0.001^{*}$ \\
Flaco/Gordo & -1.336 & 486.35 & 0.182 \\
Bajo/Alto & -1.855 & 479.658 & 0.064 \\
Guapo/Feo & -1.138 & 486.42 & 0.256 \\
Chico/Grande & -2.920 & 480.099 & $0.004^{*}$ \\
Activo/Inactivo & -1.178 & 485.144 & 0.239 \\
Enfermo/Sano & 3.409 & 478.128 & $0.001^{*}$ \\
\hline *Sig.<.01 & & &
\end{tabular}

Tabla VIII. Resultados prueba t para autoconcepto académico entre niños y niñas

\begin{tabular}{lccc}
\hline $\begin{array}{l}\text { Autoconcepto } \\
\text { Académico }\end{array}$ & $t$ & $g l$ & $\begin{array}{c}\text { Sig. } \\
\text { (bilateral) }\end{array}$ \\
\hline Estudioso/Flojo & 3.208 & 459.923 & $0.001^{*}$ \\
Rápido/Lento & -0.482 & 478.847 & 0.630 \\
Tonto/Listo & -1.220 & 310.855 & 0.224 \\
Bueno/Malo & 1.262 & 469.709 & 0.208 \\
Burro/Aplicado & -1.19 & 475.952 & 0.235 \\
Cumplido/Incumplido & 0.488 & 485.727 & 0.626 \\
Flojo/trabajador & -3.347 & 441.147 & $0.001 *$ \\
Organizado/Desorganizado & 1.932 & 470.864 & $0.054^{* *}$ \\
Atrasado/Adelantado & -0.206 & 471.786 & 0.837 \\
\hline *Sig.<.01;***Sig.<.05 & & &
\end{tabular}

\subsection{Análisis de conglomerados}

Para analizar la existencia de conglomerados de estudiantes con características distintivas, de acuerdo con sus percepciones de alimentación, actividad física y preocupación por la obesidad y la apariencia física, se realizó un análisis de conglomerados en dos fases, el cual ofrece la posibilidad de maximizar las diferencias inter-grupo y establecer automáticamente el número más apropiado de conglomerados (Martín y de Paz, 2007).

Se identificaron dos conglomerados de participantes, diferenciados en su nivel según la globalidad de las respuestas a los diferentes cuestionamientos y por el número de participantes que hay en ellos (ver figura 1). En la tabla IX se muestra la distribución de los estudiantes en los dos conglomerados, de acuerdo con el nivel de importancia del predictor (de mayor a menor). Como se observa en la tabla, el primer conglomerado ( $40.7 \%$ de sujetos) se distingue por aglutinar estudiantes que en una buena proporción considera que el valor nutritivo de los productos que venden en la tienda escolar es malo (50.3\%) y estos productos son malos para la salud (70.2\%); $36.1 \%$ compra algunos días en la tienda escolar y $36.7 \%$ sólo va una vez al día; $78.9 \%$ desayuna en casa, $60.8 \%$ trae lunch de casa y $82.5 \%$ cree que la apariencia física importa.

El segundo conglomerado (59.3\% de sujetos) se diferencia porque el 59\% de los estudiantes considera que el valor nutritivo de los productos que venden en la tienda escolar no es tan bueno, pero $60.2 \%$ no sabe si estos productos son malos para la salud, $53.4 \%$ compra algunos días en la tienda escolar y $67.5 \%$ sólo va una vez al día, el $94.4 \%$ desayuna en casa, $78.7 \%$ trae lunch de casa y $75.1 \%$ cree que la apariencia física importa. 


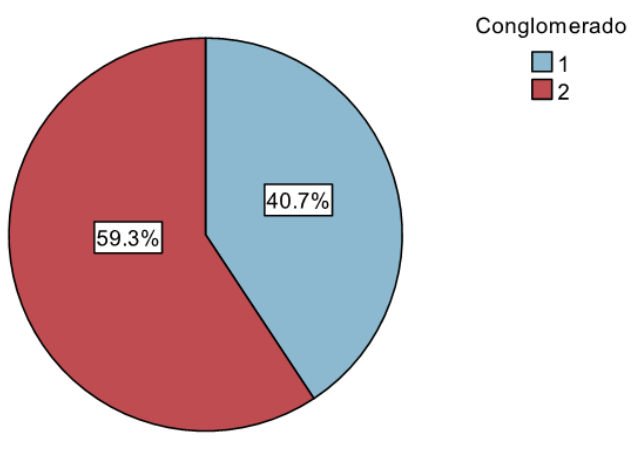

Figura 1. Distribución de los estudiantes en dos conglomerados generados a partir de sus percepciones de alimentación, actividad física y preocupación por la obesidad y apariencia física

Tabla IX. Distribución de los estudiantes de acuerdo con el nivel de importancia del predictor

\begin{tabular}{|c|c|c|c|c|}
\hline \multirow{2}{*}{ Descriptor } & \multicolumn{4}{|c|}{ Conglomerado } \\
\hline & \multicolumn{2}{|l|}{1} & \multicolumn{2}{|l|}{2} \\
\hline $\begin{array}{l}\text { Percepción del valor nutritivo de los productos que } \\
\text { venden en la tienda escolar }\end{array}$ & Malo & $50.3 \%$ & No tan bueno & $59 \%$ \\
\hline $\begin{array}{l}\text { Calificación del nivel nutricional de los productos que } \\
\text { venden en la tienda escolar }\end{array}$ & $\begin{array}{l}\text { Para nada } \\
\text { nutritivo }\end{array}$ & $45 \%$ & $\begin{array}{l}\text { Un poco } \\
\text { nutritivo }\end{array}$ & $51 \%$ \\
\hline $\begin{array}{l}\text { Percepción de los productos que se venden en la tienda } \\
\text { escolar }\end{array}$ & $\begin{array}{l}\text { Malos para la } \\
\text { salud }\end{array}$ & $70.2 \%$ & No sé & $60.2 \%$ \\
\hline Veces al día que compra en la tienda de la escuela & Una vez al día & $35.7 \%$ & Una vez al día & $67.5 \%$ \\
\hline Días a la semana que compra en la tienda escolar & Algunos días & $35.1 \%$ & Algunos días & $53.4 \%$ \\
\hline $\begin{array}{l}\text { Importancia de los productos que vende la tienda en una } \\
\text { dieta sana }\end{array}$ & $\begin{array}{l}\text { Para nada } \\
\text { importante }\end{array}$ & $36.1 \%$ & $\begin{array}{l}\text { No muy } \\
\text { importante }\end{array}$ & $33.3 \%$ \\
\hline Normalmente desayuna en casa & Sí & $78.9 \%$ & Sí & $94.4 \%$ \\
\hline La escuela cuenta con bebederos o garrafones de agua & No & $67.3 \%$ & No & $85.9 \%$ \\
\hline Normalmente trae lunch de casa & Sí & $60.8 \%$ & Sí & $78.7 \%$ \\
\hline Compra más de dos veces a la semana en la tienda escolar & Sí & $43.3 \%$ & Sí & $45.4 \%$ \\
\hline Considera que la apariencia física importa & Sí & $82.5 \%$ & Sí & $75.1 \%$ \\
\hline $\begin{array}{l}\text { Sin contar la clase de educación física, hace más ejercicio } \\
\text { dentro y fuera de la escuela }\end{array}$ & Sí & $93 \%$ & Sí & $92.8 \%$ \\
\hline
\end{tabular}

\section{Discusión y conclusiones}

A pesar de que en las zonas de marginación en México se carece de los beneficios del desarrollo, el acceso a los bienes y servicios básicos vinculados a educación, vivienda e ingresos (Cortés, 2002), los resultados evidencian aspectos positivos relacionados con la talla y peso promedio tanto de los niños como de las niñas, así como la preocupación desde el hogar para que los niños lleven a la escuela alimentos preparados para comer en ella y el acceso en un par de escuelas al programa gubernamental de "desayunos escolares calientes" que contribuye a mejorar el estado nutricional de los escolares. Aunque prevalece la necesidad de la adecuación de la dieta infantil debido al consumo de "productos chatarra" que se adquieren en las propias tiendas o puestos escolares, lo que coloca a los niños entre la obesidad y la desnutrición vinculada al exceso de consumo de carbohidratos, bebidas industrializadas y en general a una alimentación complementaria de mala calidad.

Más allá de lo que se consume, los resultados evidencian bajo autoconcepto físico y académico tanto en niños como en niñas, lo que hace susceptible a esta población a la estigmatización y a un mayor riesgo de presentar dificultades en sus relaciones familiares y sociales, trastornos alimenticios, ansiedad y obtención de un bajo rendimiento escolar (García, Musitu y Veiga, 2006; Marsh y Craven, 2006; Willows et al., 2013). 
Aunque los resultados son bajos en los atributos del autoconcepto físico, las niñas presentan significativamente mejores resultados que los niños, lo que difiere de los resultados de otros estudios en otros contextos en los que los niños presentaron un autoconcepto físico más positivo (Lau, 1990; Hay y Ashman, 2003, Hergovich, Sirsch y Felinger, 2004). También existe un bajo autoconcepto académico, pero se encontraron diferencias significativas más favorables en los niños respecto de las niñas, difiriendo también de los resultados en otros contextos (Cains et al., 1990). El autoconcepto se vuelve más diferenciado con la edad, disminuyendo a principios de la adolescencia y aumentando en la etapa adulta (Marsh, 1989). De ahí que la etapa de la pre-adolescencia es clave para reforzar el autoconcepto físico el cual suele decaer en la etapa adolescente.

Dos perfiles de estudiantes se encontraron, uno de ellos puede describirse como más consciente del valor nutricional de los productos que consumen en la tienda escolar y otro de ellos que puede catalogarse como menos consciente tanto del valor como del nivel nutritivo de los productos alimenticios a los que tienen acceso en la escuela, aunque para ambos grupos es relevante la imagen corporal y la realización de ejercicio adicional al que se realiza en la clase de Educación Física.

A partir de los hallazgos de este estudio consideramos que es necesario desarrollar estrategias de intervención que fortalezcan ambas dimensiones del autoconcepto en los escolares en esta etapa de su formación, ya que un autoconcepto físico positivo contribuye al bienestar y a la regulación emocional, fortaleciendo las redes de socialización. Aunado a que -bajo las condiciones de marginación en el entorno- el reforzamiento del autoconcepto académico en los niños puede funcionar como un mecanismo motivacional para lograr un mejor desempeño académico (Chong, 2007; González et al., 2012), así como la continuidad de los estudios hacia la secundaria y con ello reducir la tasa de deserción escolar. Dado que los hábitos saludables adquiridos durante la infancia y adolescencia tienden a mantenerse en la edad adulta y los grupos en edad escolar básica suelen presentar autoconceptos bajos y años después es cuando se presentan mejoras (Brownell et al., 2007; Marsh, 1989), es recomendable que se realicen estudios longitudinales en contextos de marginación que permitan identificar cómo inciden las intervenciones a lo largo del tiempo en las dimensiones del autoconcepto, el rendimiento escolar y otras variables de bienestar personal que pudieran guiar programas educativos que contribuyan a un desarrollo integral de los niños y jóvenes.

\section{Referencias}

Acosta, M. V., y Goméz, G. (2003). Insatisfacción corporal y seguimiento de dieta. Una comparación transcultural entre adolescentes de España y México. Revista Internacional de Psicología Clínica y de la Salud, 3(1), 9-21.

Alexis, A. (2014). Estudio comparativo del autoconcepto en niños prescolares en situaciones de pobreza y en ambientes más favorecidos. Entretemas, 12, 41-62.

Amar, A. (2000). Niños invulnerables. Factores cotidianos de protección que favorecen el desarrollo de los niños que viven en contextos de pobreza. Psicología desde el Caribe, 5, 96-126.

Andrade, P., y Pick, S. (1986). Una escala de autoconcepto para niños. La psicología social en México. México: Asociación Mexicana de Psicología Social.

Bailón, C. (2013). Ideología mediática y educación en México, ¿influyen en la construcción y mantenimiento de la desigualdad social? Teoría y crítica de la psicología, 3, 20-29.

Bolognini, M., Plancherel, B., Bettschart, W., y Halfon, O. (1996). Self-esteem and mental health in early adolescence: development and gender differences. Journal of Adolescence, 19(3), 233-245.

Brownell, C., Zerwas, S., y Ramani, G.B. (2007). "So big": The development of body self-awareness in toddlers. Child Development, 78(5), 1426-1440. 
Cairns, E., McWhirter, L., Duffy, U., y Barry, R. (1990). The stability of self- concept in late adolescence: gender and situational effects. Personality and Individual Differences, 11(9), 937-944.

Chong, W. H. (2007). The role of personal agency beliefs in academic self-regulation: an Asian perspective. School Psychology International, 28(1), 63-76.

Cortés, F. (2002). Consideraciones sobre la marginalidad, marginación, pobreza y desigualdad en la distribución del ingreso. Papeles de Población, 8(31), 9-24.

Costa, M. C. V. (Org.) (2009). A educação na cultura da mídia e do consumo. Río de Janeiro: Lamparina.

Fox, K. R., y Corbin, C. B. (1989). The Physical self-perception profile: development and preliminary validation. Journal of Sport and Exercise Psychology, 11, 408-433.

Franco, C. (2006). Relación entre las variables autoconcepto y creatividad en una muestra de alumnos de educación infantil. Revista Electrónica de Investigación Educativa, 8(1), 1-16. Recuperado de http://redie.uabc.mx/redie/article/view/120

García, J. F., Musitu, G., y Veiga, F. (2006). Autoconcepto en adultos de España y Portugal. Psicothema, 18(3), 551-556.

Gosling, R., Stanistreet, D., y Swami, V. (2008). "If Michael Owen drinks it, why can't I?" -- 9 and 10 year olds' perceptions of physical activity and healthy eating. Health Education Journal, 67(3), 167-181.

González, M. L., Leal, D., Segovia, C., y Arancibia, V. (2012). Autoconcepto y talento: una relación que favorece el logro académico. Psykhe, 21(1), 37-53.

Goñi, A., Ruíz de Azúa, S., y Rodríguez, A. (2011). Deporte y autoconcepto físico en la preadolescencia. Ciencias aplicadas a la actividad física y el deporte, 77, 18-24.

Guido, P., Mújica, A., y Gutiérrez, R. (2011). Diferencias en el autoconcepto por sexo en la adolescencia: construcción y validación de un instrumento. Liberabit, 17, 139-146.

Guillén, F. (2003). Psicopedagogía de la de la actividad física y el deporte. Armenia, Colombia: Kinesis.

Hay, I., y Ashman, A. F. (2003). The development of adolescents' emotional stability and general selfconcept: the Interplay of parents, peers, and gender. International Journal of Disability, Development and Education, 50(1), 77-91.

Hergovich, A., Sirsch, U., y Felinger, M. (2004). Gender differences in the self-concept of preadolescent children. School Psychology International, 25(2), 207-222.

Kerlinger, F. N. y Lee, H. B. (2002). Investigación del comportamiento. México: McGraw-Hill.

Lau, S. (1990). Crisis and Vulnerability in Adolescent development. Journal of youth and adolescence, 19(2), 111-131.

Marsh, H. W. (1989). Age and Sex effects in multiple dimensions of self-concept: pre-adolescence to early adulthood. Journal of Educational Psychology, 81, 417-430.

Marsh, H. W., y Craven, R. G. (2006). Reciprocal effects of self-concept and performance from a multidimensional perspective: Beyond seductive pleasure and unidimensional perspectives. Perspectives on Psychological Science, 1, 133-163.

Martín, Q. y de Paz, Y. (2007). Tratamiento estadístico de datos con SPSS. México: Paraninfo. 
Mboya, M. M. (1999). Multiple Dimensions of adolescent self-concept: relations with age, gender and scholastic measures. School Psychology International, 20(4), 388-398.

Padilla, M., García, S. y Suárez, M. (2010). Diferencias de género en el autoconcepto general y académico de estudiantes de 40. de ESO. Revista de Educación, 352, 495-515.

Páez, S. (2012). La Resurrección y Santa María Xonacatepec entre colonias más pobres. Recuperado de http://www.retodiario.com/despliegue-noticia2012.php?id=35444

Ramírez, M., Devia, R. E., y León, R. A. (2011). Pobreza y rendimiento escolar: estudio de caso de jóvenes de alto rendimiento. Educere, Investigación Arbitrada, 15(52), 663-672.

Salum-Fares, A., Marín, R. y Reyes, C. (2011). Relevancia de las dimensiones del autoconcepto en estudiantes de escuelas secundarias de Ciudad Victoria, Tamaulipas, México. Revista Electrónica de Psciología Iztacala, 14(2), 255-272.

Simms, K., Bock, S. y Hackett, L. (2013). Do the duration and frequency of physical education predict academic achievement, self-concept, social skills, food consumption, and body mass index? Health Education Journal. Recuperado de http://citeseerx.ist.psu.edu/viewdoc/download?doi=10.1.1.927.3398\&rep=rep1\&type=pdf

Tatlow-Golden, M. y Guerin, S. (2010). Aspects of children's self-concept 'My favorite things to do and my favorite people': Exploring salient aspects of children's self-concept. Childhood, 17(4), 545-562.

Torres, A. y Rodrigo, M. J. (2014). La influencia del apego y el autoconcepto en los problemas de comportamiento de los niños y niñas de familias en desventaja socioeconomica. Educatio Siglo XXI, 32(2), 255-278.

Yeung, A., Craven, R. y Ali, J. (2013). Self-concepts and educational outcomes of indigenous Australian students in urban and rural school settings. School Psychology International, 34(4), 405-427.

Willows, N.D., Ridley, D., Eaine, K.D., y Maximova, K. (2013). High adiposity is associated cross-sectionally with low self-concept and body size dissatisfaction among indigenous Cree schoolchildren in Canada. BioMed Central Pediatrics, 13(118), 1-18. 